\title{
A Deep Reinforcement Learning Approach to IRS-assisted MU- MIMO Communication Systems
}

This paper was downloaded from TechRxiv (https://www.techrxiv.org).

\section{LICENSE}

CC BY 4.0

SUBMISSION DATE / POSTED DATE

04-01-2022 / 07-01-2022

\section{CITATION}

Pereira-Ruisánchez, Dariel; Fresnedo, Óscar; Pérez-Adán, Darian; Castedo, Luis (2022): A Deep Reinforcement Learning Approach to IRS-assisted MU-MIMO Communication Systems. TechRxiv. Preprint. https://doi.org/10.36227/techrxiv.17868425.v1

$\mathrm{DOI}$ 


\title{
A Deep Reinforcement Learning Approach to IRS-assisted MU-MIMO Communication Systems
}

\author{
Dariel Pereira-Ruisánchez, Student Member, IEEE, Óscar Fresnedo, Member, IEEE, Darian Pérez-Adán \\ and Luis Castedo, Senior Member, IEEE.
}

\begin{abstract}
The combination of multiple-input multiple-output (MIMO) communications and intelligent reflecting surfaces (IRSs) is foreseen as a key enabler of beyond 5G (B5G) and 6G systems. In this work, we develop an innovative deep reinforcement learning (DRL)-based approach to the joint optimization of the MIMO precoders and the IRS phase-shift matrices that is proved to be efficient in high dimensional systems. The proposed approach is termed deep deterministic policy gradient (DDPG) and maximizes the sum rate of an IRS-assisted multi-stream (MS) multi-user MIMO (MU-MIMO) system by learning the best matrix configuration through online trial-and-error interactions. The proposed approach is formulated in terms of continuous state and action spaces, and a sum-rate-based reward function. The computational complexity is reduced by using artificial neural networks (ANNs) for function approximations and it is shown that the proposed solution scales better than other state-of-theart methods, while reaching a competitive performance.
\end{abstract}

Index Terms-DRL, DDPG, MU-MIMO, intelligent reflecting surfaces.

\section{INTRODUCTION}

$\mathbf{T}$ HE research interest in intelligent reflecting surface (IRS)-assisted multiple-input multiple-output (MIMO) communication systems is increasing. By properly designing the MIMO precoding/combining matrices and the IRS phaseshift matrix, the demanding requirements of the use cases in beyond 5G (B5G) and sixth generation (6G) systems are expected to be fulfilled [1]-[6].

In [7], the use of IRSs at boundaries among multiple cells to assist the downlink transmission to cell-edge users is considered. The results show that inter-cell interference can be alleviated by destructively combining the interference signals impinging in the IRS elements. In [8], the authors analyze the deployment of IRS-assisted multi-user MIMO (MU-MIMO) systems as an energy efficient alternative to multi-antenna amplify-and-forward relaying. Numerical results show that the IRS-based approach provides up to $300 \%$ higher energy efficiency (EE) while reaching near optimal spectrum efficiency (SE). Authors in [9] address the SE performance of an IRSassisted multi-user multiple-input single-output (MU-MISO) system in a scenario with no natural line-of-sight (LoS). As expected, the sum-rate values remarkably improve when considering the base station (BS)-IRS-users links.

Several other works on IRS-assisted communications focus on industry-specific applications such as unmanned aerial vehicles (UAVs) [10], [11], physical layer security [1], [12], [13], mobile edge computing (MEC) [14]-[16], and Internet of Things (IoT) [14], [17]. Beyond this, IRS-assisted communications are being addressed as a main enabler for $6 \mathrm{G}$
Terahertz communications, although several challenges remain to be solved [18], [19].

MIMO and IRS technologies face a similar limitation since their performance and deployment complexity both increase with the number of elements. In MIMO communications, the multiplexing gain, the spatial diversity and the favorable propagation conditions can be improved by using more antennas [20]. In IRS-assisted systems, the average received signal-tonoise ratio (SNR) and the transmission power savings are directly related to the square of the number of scattering elements [1].

Several approaches have been proposed to deal with the complexity of the joint design of the MIMO precoding matrices and the IRS phase-shift matrix in IRS-assisted MIMO communications. Most of these optimization problems are non-convex and non-polynomial time solvable because of the form of the power/rate constraints and the high number of elements to optimize. Conventional approaches relax the original problem, approximate non-convex constraints iteratively, or change the domain of the optimization, sacrificing optimality for the sake of tractability. Metaheuristic and genetic optimization algorithms find near optimal solutions by using less computationally complex operations, but their slow convergence can be a major limitation when dealing with timevarying channels and network configurations [3], [21]-[26].

In this work we will consider deep learning (DL) to solve optimization problems like these where classical solutions become too complex or the search spaces are too vast. DL is a machine learning paradigm where input and output features are related through mathematical blocks of weighted connections and their activation functions, namely artificial neural networks (ANNs). The weights of the ANNs are trained, mainly by means of gradient descent-based algorithms, to learn and later generalize the actual correspondence between the input and output sets. Several works have proposed the use of supervised DL approaches to solve different challenging problems in IRS-assisted MIMO communications like channel estimation [27]-[33], beamforming [34], [35] and IRS phase-shift matrix optimization [36]. However, the performance of supervised DL approaches is highly dependent on the data sets available for the training process. The supervised training requires large amount of labeled data (i.e., input sets and their corresponding output sets), being the lack of realistic training data one of the main limitations for this strategy. Furthermore, the training and the operational data must have the same distribution to ensure a good performance of the trained models. Hence, predictions are very sensitive to modifications in the communication 
system or in the configuration parameters [37].

Due to the limitations of the aforementioned solutions, we propose instead the use of deep reinforcement learning (DRL) to solve the joint optimization of the precoding and IRS phase-shift matrices in an IRS-assisted multi-stream (MS) MU-MIMO uplink. Conventional reinforcement learning (RL) and DRL are two branches within machine learning. DRL, like supervised DL, exploits the predictive capability of the ANNs to emulate complex systems. However, unlike supervised learning, the DRL model is trained in an online fashion by interacting with the communication system itself. Hence, DRL models do not require neither previous offline training nor labeled sets of data. DRL-based solutions have been employed in different applications of wireless communication systems, proving to be an appealing alternative when tackling complex optimization problems [38]-[45].

To the best of our knowledge, DRL has not been previously considered to solve the optimization problem we are dealing with. In this sense, the main contributions of the paper are summarized as:

- We propose the use of the DRL-based deep deterministic policy gradient (DDPG) framework to optimize the user precoding matrices and the IRS phase-shift matrix in order to maximize the sum-rate of an IRS-assisted MS MU-MIMO uplink communication.

- We assess the performance of the proposed framework in several single-stream (SS) and MS MU-MIMO scenarios and the results show that our approach reaches a competitive performance in an efficient manner.

The remainder of this paper is structured as follows. Section II details some theoretical fundamentals about DRL and related works. Section III presents the system model and the formulation of the optimization problem. In Section IV, we explain our proposed solution. Section V is devoted to the validation results and Section VI presents the conclusions.

\section{Preliminaries}

This section is intended to introduce some theoretical fundamentals about the RL framework. To better clarify these concepts, we briefly analyze how some related works approach similar optimization problems.

\section{A. Reinforcement Learning Fundamentals}

As stated in [46], RL is a computational approach to learning from interaction, i.e., how to map situations to actions that maximize a numerical reward function. Trial-and-error search and delayed reward are the two most important distinguishing features of RL.

Fig. 1 shows the main components of the RL framework and how they interact. The agent is the learning element in the framework and it aims at learning the best action $a_{t}$ at instant $t$ for the current state $s_{t}$. The optimality of the action $a_{t}$ is related to the value of the instantaneous reward $r_{t}$ that the agent receives by taking $a_{t}$ while being in $s_{t}$, and the expected rewards in future states. The environment is the external element which the agent interacts with. The environment controls the value of $r_{t}$ given $s_{t}$ and $a_{t}$, and

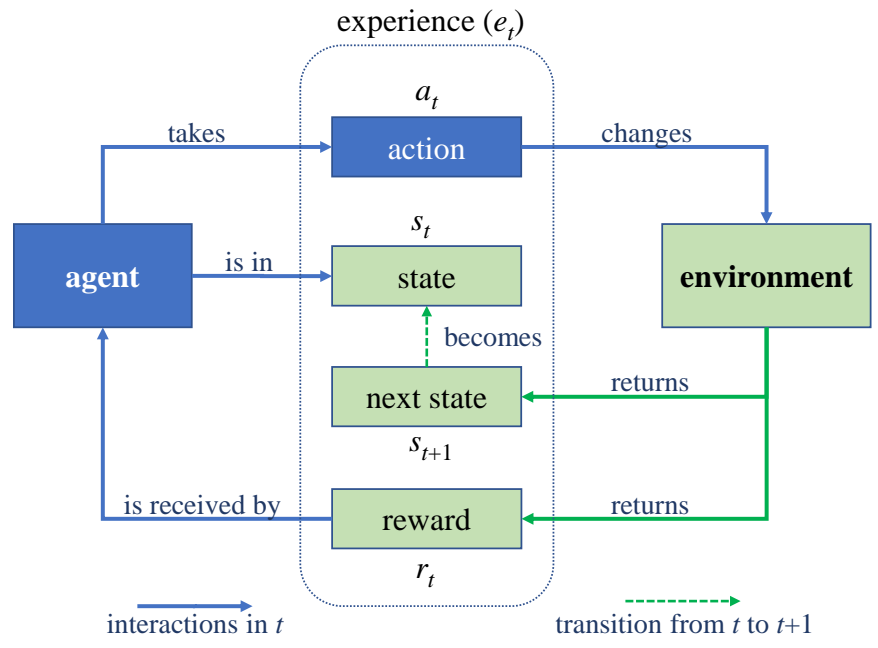

Fig. 1. RL framework.

defines the next state $s_{t+1}$ where the agent will be. The tuple $e_{t}=\left\{s_{t}, a_{t}, r_{t}, s_{t+1}\right\}$ is commonly termed experience.

Another important element of the RL framework is the policy. The policy is the decision-making rule that defines the action that the agent will take while being in a given state, i.e., $a_{t}=\pi\left(s_{t}\right)$, where $a_{t}$ belongs to the set $\mathcal{A}\left(s_{t}\right)$ of actions available in $s_{t}$, and $\pi(\cdot)$ is a deterministic policy. The goal of RL algorithms can be summarized as to find the optimal policy $\pi^{*}(\cdot)$ that maximizes a function of the long-term reward (current and future rewards).

The action-value function $q_{\pi}(\cdot)$ is the long-term reward function that is mostly employed in RL algorithms. This is a function of $s_{t}$ and $a_{t}$ such that $q_{\pi}\left(s_{t}, a_{t}\right)=\sum_{k=0}^{\infty} \gamma^{k} r_{t+k}$, where $\gamma$ stands for a discount factor and $\pi(\cdot)$ is the policy to follow from the next state onwards. It has been proved that the optimal policy $\pi^{*}(\cdot)$ can be obtained by taking greedy actions according to the Bellman optimality equation $q_{*}\left(s_{t}, a_{t}\right)=\mathbb{E}\left[r_{t}+\gamma \max _{a_{t+1}} q_{*}\left(s_{t+1}, a_{t+1}\right)\right]$. Hence, the solution to the RL problems can be found by updating the $q_{\pi}(\cdot)$ function values in an iterative-manner until optimality is reached.

Although Bellman optimality equations provide a way to find the optimal policy, the conventional RL algorithms cannot be applied to our optimization problem. Conventional tabular methods are constrained to low-dimensional discrete state and action spaces since memory and computational requirements become unaffordable. In those problems where action and state spaces are continuous or arbitrarily large, function approximation methods must be considered for both the policy and the long-term reward functions [46]-[48].

\section{B. Deep Reinforcement Learning}

DRL-based methods cover several algorithms that use ANNs to approximate the policy and/or value functions. On the one hand, Deep Q-learning (DQL) is a DRL algorithm, mostly used to solve optimization problems with continuous state spaces and low-dimensional discrete action spaces. It learns an approximation of the $q_{\pi}(\cdot)$ function, and uses it 
TABLE I

RELATED WORKS' SUMMARY

\begin{tabular}{|c||c|l|l|c|}
\hline Reference & \multicolumn{1}{|c|}{ Scenario } & \multicolumn{1}{c|}{ State space } & \multicolumn{1}{c|}{ Action space } & Reward \\
\hline$[50]$ & IRS-assisted SU-MISO & $\begin{array}{l}\text { IRS matrix current values } \\
\text { and previous step reward }\end{array}$ & IRS matrix next values & SNR \\
\hline [51] & IRS-assisted MU-MISO & $\begin{array}{l}\text { IRS matrix current values, } \\
\text { precoding matrix current } \\
\text { values, CSI and power- } \\
\text { related values }\end{array}$ & $\begin{array}{l}\text { IRS matrix next values } \\
\text { and precoding matrix next } \\
\text { values }\end{array}$ & sum-rate \\
\hline
\end{tabular}

to infer the optimal policy. This algorithm has been widely employed in wireless communications to tackle large combinatorial problems like those in resource allocation [38], [49]. However, it cannot handle high-dimensional/continuous action space problems.

On the other hand, the policy gradient (PG) DRL-based algorithms have been recently addressed as a solution to RL problems with continuous state and action spaces. In these methods, the policy function is also parameterized by exploiting the generalization capability of the ANNs.

PG DRL-based methods circumvent most of the limitations of conventional RL. Because of this, we propose the use of the DDPG framework to handle our optimization problem. Some of the features that support our choice are:

- deterministic: each state is mapped into one specific action. This mapping is expected to converge to the actions that maximize the long-term reward function.

- model-free learning: knowing the environment dynamics is not necessary for learning. The algorithm learns only through the interactions.

- off-policy: exploration policy can be treated independently from the target policy. Samples for training can be obtained by following any policy and the learning capability of the target policy is not affected [46]-[48]. On the other hand, on-policy algorithms employ the same policy in both stages, thus reducing their exploratory capability.

\section{Related Works}

In this subsection, we will see how different approaches to the same DDPG framework have been used to solve similar optimization problems in MISO communications. Table I shows the differences and similarities between the elements that authors included in the state and action spaces, and the reward functions.

In [50], the authors propose to use the DDPG framework to optimize the phase-shift matrix in an IRS-assisted singleuser MISO (SU-MISO) system. They propose to employ the current values of the IRS phase-shifts and the value of the reward received in the previous state as the elements of the current state. The action is composed of the next values of the IRS phase shifts, and the reward is the value of the SNR perceived by the user if these IRS phase shifts were used. Apparently, with this configuration the rewards depend only on the actions. Hence, the same reward will be received in any state as long as the same action is taken.

Authors in [51] propose to use this framework to solve the joint optimization of the IRS matrix and the precoding matrix in a MU-MISO downlink communication. As in [50], they include the current entries of the different matrices as part of the state (IRS phase-shift matrix and precoding matrix). Additionally, several elements of the channel state information (CSI), the received power and the transmitted power are included in the state. By adding these elements, the dimension of the state space significantly increases but the extra information could improve the learning capability of the networks. The action is composed of the next values of the IRS and precoding matrices. The instantaneous sum-rate achieved with the action matrices is the reward. Authors in [51] explain that the real and imaginary parts of complex-valued actions and states must be treated as independent inputs, since conventional ANNs can only handle real-valued inputs. On the other hand, complex-valued networks might have been considered in [50], since the real and imaginary parts are not individually handled [28].

Due to the fact that the next values for the optimized matrices are being considered as the actions, authors in [51] include a projection stage between the policy and the actionvalue functions. This stage projects the output of the policy function approximation into feasible matrices that meet the power constraint and the unitary-modulus constraint for the precoding matrix and the IRS matrix, respectively. This stage increases the computational complexity of the learning process, since calculating the gradients at this stage can be a cumbersome process.

Results in [50] and [51] support that the DDPG framework is an appealing alternative for this kind of optimization problems, even by considering different approaches to the action and state spaces.

\section{System Model And Optimization Problem}

\section{A. Notation}

Along this work, the following notation is employed: $a$ is a scalar, $\mathbf{a}$ is a vector, and $\mathbf{A}$ is a matrix. Transpose and conjugate transpose of $\mathbf{A}$ are represented by $\mathbf{A}^{T}$ and $\mathbf{A}^{H}$, respectively. $\|\mathbf{A}\|_{F}^{2}$ and $\operatorname{tr}[\mathbf{A}]$ denote the Frobenius norm and the trace of $\mathbf{A}$, respectively. $\mathbf{I}_{N}$ indicates an $N \times N$ identity matrix, $\mathcal{I}_{N}$ denotes the set of integers from 1 to $N$. The operator blkdiag $(\cdot)$ constructs a block diagonal matrix from its input matrices. Finally, expectation is represented by $\mathbb{E}[\cdot]$.

\section{B. IRS-assisted MS MU-MIMO Uplink}

Let us consider the uplink of an IRS-assisted MS MU-MIMO communication system as shown in Fig. 2. In this scenario, each of the $K$ users employs $N_{\mathrm{t}}$ antennas to send $N_{\mathrm{s}}$ 


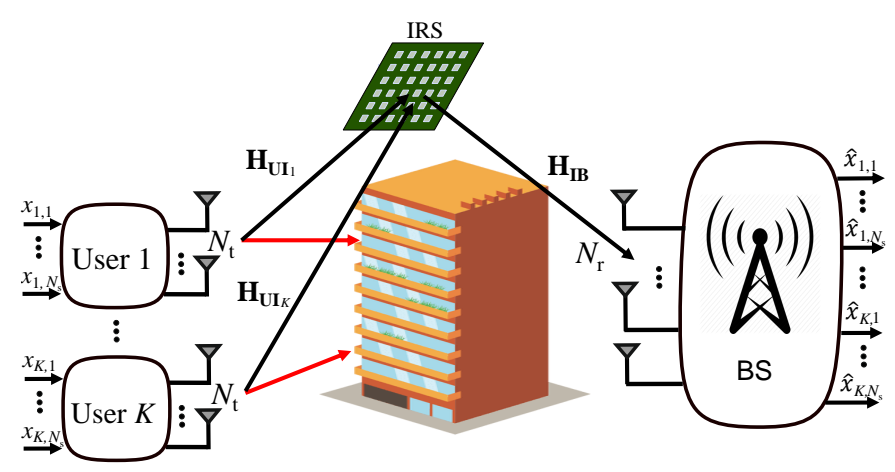

Fig. 2. Uplink of an IRS-assisted MS MU-MIMO system.

data streams to a BS equipped with $N_{\mathrm{r}}$ antennas. We assume there is total blockage between the users and the BS, i.e., there is no direct channel available between any of the $K$ users and the BS.

The symbols transmitted by the $k$-th user on each channel usage are represented by $\mathbf{x}_{k}=\left[x_{k, 1}, \ldots, x_{k, N_{\mathrm{s}}}\right]^{T} \in \mathbb{C}^{N_{\mathrm{s}} \times 1}$. We assume $\mathbf{x}_{k}$ follows a zero-mean multivariate complexvalued Gaussian distribution, i.e., $\mathbf{x}_{k} \sim \mathcal{N}_{\mathbb{C}}\left(0, \mathbf{I}_{N_{\mathrm{s}}}\right)$. Such symbols are linearly precoded with the precoding matrix $\mathbf{P}_{k} \in \mathbb{C}^{N_{\mathrm{t}} \times N_{\mathrm{s}}}$. The power transmitted by the $k$-th user is limited to $T_{k}$ which leads to the individual power constraint on the $k$-th user precoding matrix $\left\|\mathbf{P}_{k}\right\|_{F}^{2} \leq T_{k}, \forall k$.

The deployed IRS is assumed to have $N$ discrete elements. Thus, the IRS phase-shift matrix is represented by the diagonal matrix $\boldsymbol{\Theta}=\operatorname{diag}\left(e^{j \theta_{1}}, \ldots, e^{j \theta_{N}}\right) \in \mathcal{D}$ where $\theta_{n} \in[0,2 \pi)$ represents the phase shift introduced by the $n$-th element of the IRS. $\mathcal{D} \in \mathbb{C}^{N \times N}$ is the set of feasible IRS matrices i.e., the set of diagonal matrices with unit modulus entries in the main diagonals. The vector that contains the elements in the main diagonal of $\Theta$ is $\boldsymbol{\theta} \in \mathbb{C}^{N \times 1}$.

According to this system model, the received signal at the $\mathrm{BS}$ is

$$
\mathbf{y}=\mathbf{H}_{\mathrm{IB}} \Theta \sum_{k=1}^{K} \mathbf{H}_{\mathrm{UI} k} \mathbf{P}_{k} \mathbf{x}_{k}+\mathbf{n},
$$

where the matrix $\mathbf{H}_{\mathrm{UI} k} \in \mathbb{C}^{N \times N_{\mathrm{t}}}$ represents the channel response of the link from the $k$-th user to the IRS, $\mathbf{H}_{\mathrm{IB}} \in \mathbb{C}^{N_{\mathrm{r}} \times N}$ stands for the channel response from the IRS to the BS, and $\mathbf{n}=\left[n_{1}, n_{2}, \ldots, n_{N_{\mathrm{r}}}\right]^{T}$ represents the complex-valued additive white Gaussian noise (AWGN) which is modeled as $\mathbf{n} \sim \mathcal{N}_{\mathbb{C}}\left(0, \sigma_{\mathrm{n}}^{2} \mathbf{I}_{N_{\mathrm{r}}}\right)$.

Using a more compact notation, the received signal at the BS given by (1) can be rewritten as

$$
\mathbf{y}=\mathbf{H}_{\mathrm{IB}} \Theta \mathbf{H P x}+\mathbf{n},
$$

where $\mathbf{H}=\left[\mathbf{H}_{\mathrm{UI} 1}, \ldots, \mathbf{H}_{\mathrm{UI} K}\right], \mathbf{x}=\left[\mathbf{x}_{1}^{T}, \ldots, \mathbf{x}_{K}^{T}\right]^{T} \in$ $\mathbb{C}^{K N_{\mathrm{s}} \times 1}$ and $\mathbf{P}=\operatorname{blkdiag}\left(\mathbf{P}_{1}, \ldots, \mathbf{P}_{K}\right) \in \mathcal{P}$ represents the block-diagonal matrix which stacks all the user precoders. $\mathcal{P} \in \mathbb{C}^{N_{\mathrm{t}} K \times N_{\mathrm{s}} K}$ is the set of block-diagonal matrices with Frobenius norm-constrained submatrices.

The vector with all the estimated user symbols $\hat{\mathbf{x}}=$ $\left[\hat{\mathbf{x}}_{1}^{T}, \ldots, \hat{\mathbf{x}}_{K}^{T}\right]^{T} \in \mathbb{C}^{K N_{\mathrm{s}} \times 1}$ is obtained by linear filtering of the signal received at the BS, i.e., $\hat{\mathbf{x}}=\mathbf{W}^{H} \mathbf{y}$, being $\mathbf{W}^{H}=\left[\mathbf{W}_{1}, \ldots, \mathbf{W}_{K}\right]^{H} \in \mathbb{C}^{K N_{\mathrm{s}} \times N_{\mathrm{r}}}$ the BS receiving filter matrix that stacks the user individual receiving filter matrices $\mathbf{W}_{k}^{H} \in \mathbb{C}^{N_{\mathrm{s}} \times N_{\mathrm{r}}}$. We assume $\mathbf{W}_{k}^{H}, \forall k$ to be the minimum mean square error (MMSE) receiving filters given by

$$
\begin{aligned}
& \mathbf{W}_{k}^{H}=\mathbf{P}_{k}^{H} \mathbf{H}_{k}^{H} \mathbf{\Theta}^{H} \mathbf{H}_{\mathrm{IB}}^{H} \\
& \times\left(\mathbf{H}_{\mathrm{IB}} \boldsymbol{\Theta}_{k} \mathbf{H}_{k} \mathbf{P}_{k}^{H} \mathbf{H}_{k}^{H} \boldsymbol{\Theta}^{H} \mathbf{H}_{\mathrm{IB}}^{H}+\sigma_{\mathrm{n}}^{2} \mathbf{I}_{N_{\mathrm{r}}}\right)^{-1} .
\end{aligned}
$$

We next look for the IRS phase-shift matrix and the user precoding matrices that maximize the achievable sum-rate. Towards this aim, we formulate the following optimization problem

$$
\begin{aligned}
& \underset{\mathbf{P}, \boldsymbol{\Theta}}{\arg \max } \sum_{k=1}^{K} R_{k} \\
& \text { s.t. }\left\|\mathbf{P}_{k}\right\|_{F}^{2} \leq T_{k}, \forall k \in \mathcal{I}_{K}, \\
& \quad \boldsymbol{\Theta} \in \mathcal{D},
\end{aligned}
$$

where $R_{k}$ is the $k$-th user individual rate which is given by

$$
\begin{aligned}
& R_{k}=\log _{2} \operatorname{det}\left(\mathbf{I}_{K}+\mathbf{X}_{k}^{-1}\right. \\
& \left.\times \mathbf{W}_{k}^{H} \mathbf{H}_{\mathrm{IB}} \boldsymbol{\Theta} \mathbf{H}_{\mathrm{UI} k} \mathbf{P}_{k} \mathbf{P}_{k}^{H} \mathbf{H}_{\mathrm{UI} k}^{H} \Theta^{H} \mathbf{H}_{\mathrm{IB}}^{H} \mathbf{W}_{k}\right),
\end{aligned}
$$

where

$$
\begin{aligned}
& \mathbf{X}_{k}=\sum_{i \neq k} \mathbf{W}_{k}^{H} \mathbf{H}_{\mathrm{IB}} \boldsymbol{\Theta} \mathbf{H}_{\mathrm{UI} i} \mathbf{P}_{i} \mathbf{P}_{i}^{H} \mathbf{H}_{\mathrm{UI} i}^{H} \Theta^{H} \mathbf{H}_{\mathrm{IB}}^{H} \mathbf{W}_{k} \\
& +\sigma_{\mathrm{n}}^{2} \mathbf{W}_{k}^{H} \mathbf{W}_{k}
\end{aligned}
$$

is the interference plus noise matrix and $\mathbf{W}_{k}^{H}$ is the MMSE individual receiving filter for the $k$-th user (3). Notice that for the SS scenarios, $N_{\mathrm{s}}=1$ and the user individual receiving filter matrices $\mathbf{W}_{k}^{H} \in \mathbb{C}^{N_{\mathrm{s}} \times N_{\mathrm{r}}}$ will be vectors $\mathbf{w}_{k}^{H} \in \mathbb{C}^{1 \times N_{\mathrm{r}}}$ instead.

As stated in (4), the user precoding matrices must meet the individual power constraints $\left\|\mathbf{P}_{k}\right\|_{F}^{2} \leq T_{k}, \forall k$. For the sake of simplicity, we assume them to be equal, i.e., $T_{k}=T, \forall k$. Without loss of generality, we also assume the noise variance $\sigma_{\mathrm{n}}^{2}$ to be equal to one, and therefore the SNR per user is given by $\mathrm{SNR}_{(\mathrm{dB})}=10 \log _{10}(T)$.

Due to the choice of the objective function and the design constrains for the IRS and the users' precoders, the problem in (4) becomes a non-convex non-trivial optimization problem. Hence, we propose to use the DRL-based DDPG framework to handle this problem in an efficient way.

\section{Channel Model}

The channel responses from the users to the IRS $(\mathbf{H}=$ $\left.\left[\mathbf{H}_{\mathrm{UI} 1}, \ldots, \mathbf{H}_{\mathrm{UI} K}\right]\right)$ and from the IRS to the $\mathrm{BS}\left(\mathbf{H}_{\mathrm{IB}}\right)$ are assumed to be perfectly known. Although channel acquisition is a difficult task in IRS-aided systems, a promising research line is committed to face this problem [52]. For $\mathbf{H}_{\mathrm{UI} k}$ channel response matrices, we assume a Rayleigh fading model, i.e., the entries of $\mathbf{H}_{\mathrm{UI} k}, \forall k$ are independent and identically distributed (i.i.d.) complex-valued circularly symmetric Gaussian random variables. On the other hand, considering that the IRS 
is typically installed to ensure $\operatorname{LoS}$ to the $\mathrm{BS}$, a Rician fading channel model is adopted to describe $\mathbf{H}_{\mathrm{IB}}$ as in [53], i.e.,

$$
\mathbf{H}_{\mathrm{IB}}=\sqrt{\frac{\beta}{1+\beta}} \mathbf{H}_{\mathrm{IB}}^{\mathrm{LOS}}+\sqrt{\frac{1}{1+\beta}} \mathbf{H}_{\mathrm{IB}}^{\mathrm{NLOS}},
$$

where $\beta$ is the Rician factor, whereas $\mathbf{H}_{\mathrm{IB}}^{\mathrm{LOS}}$ and $\mathbf{H}_{\mathrm{IB}}^{\mathrm{NLOS}}$ stand for the deterministic LoS component and the Rayleigh fading component, respectively.

\section{DDPG-BASED JOINT OPTIMIZATION}

In this section we develop a DDPG-based approach to solve (4). We start addressing the selection of the state, action and reward elements. Next, we review some important components in the DDPG framework and, finally, we derive the algorithmic solution.

\section{A. State, Action and Reward}

As shown in related works, there is not an unique valid alternative to select the elements that form the action and state spaces, and the reward in DRL. However, this selection will affect the performance of the DRL algorithm. Next, we describe our choices for each:

- State: the state vector $\mathbf{s}_{t}$ is composed of the current values of all the user precoding matrices $\mathbf{P}_{k} \in \mathbb{C}^{N_{t} \times N_{s}}$, $\forall k \in \mathcal{I}_{K}$, and the current values of the elements in the main diagonal of the IRS phase-shift matrix, i.e., in the vector $\boldsymbol{\theta} \in \mathbb{C}^{N \times 1}$. Since the real and imaginary parts of complex-valued numbers must be treated as independent inputs, the actual dimension of the state space is $D_{\text {state }}=2 K N_{\mathrm{t}} N_{\mathrm{s}}+2 N$. The state is constructed such that

$$
\mathbf{s}_{t}=\left[\text { flatten }\left(\mathbf{P}_{1}\right), \ldots, \text { flatten }\left(\mathbf{P}_{K}\right) \text {, flatten }(\boldsymbol{\theta})\right],
$$

where flatten $(\cdot)$ is the operator that transforms any matrix $\mathbf{V} \in \mathbb{C}^{A \times B}$ into a vector $\mathbf{v} \in \mathbb{C}^{1 \times 2 A B}$, by first concatenating all the real parts and later the imaginary ones. Recall that the state space is continuous since the elements of $\mathbf{s}_{t}$ can take any real value as long as the optimization constraints in (4) are met.

- Action: the action vector $\mathbf{a}_{t}$ is composed of variations in the state values such that $\mathbf{s}_{t+1}=\mathbf{s}_{t}+\mathbf{a}_{t}$. As a consequence, both the dimension and the structure of the action and the state vectors are the same. Hence, the dimension of the action space is $D_{\text {action }}=D_{\text {state }}$. The action space is also continuous although its values are constrained to the real-valued interval $(-1,1)$ in order to improve the system stability.

- Reward: since we aim at finding the matrices that maximize the sum-rate, we use this value to calculate the reward. We calculate the instantaneous reward $r_{t}$ as the difference between the sum-rates after and before taking the action $\mathbf{a}_{t}$, instead of using the sum-rate value itself.

We decided not to include additional channel information in the state because the state space dimension would increase significantly. For example, if we consider a system with $K=$ $5, N_{\mathrm{s}}=3, N_{\mathrm{t}}=5, N=50$ and $N_{\mathrm{r}}=30$, the dimension of the state space raises from 250 to 5750 elements when including the CSI. Increasing the state space dimension forces to increase also the size of the ANNs and the memory capacity of the experience replay buffer. This can be a major limitation for practical developments.

Actions, in the way we have defined them, allow us to identify a correspondence between the current state and the action taken, and the next state. Furthermore, the instantaneous reward depends on both the state and the action, and not only on the action.

ANNs are very sensitive to the scale of the features. If we use the sum-rate value itself as reward, the scale difference between the input and the output features can be unfavorable to the learning. By considering the reward to be the difference between the sum-rate values, the scales of the state, action and reward values remain similar.

\section{B. DDPG Framework Elements}

DDPG is a model-free off-policy algorithm, as described in Section II. This method was first introduced in [48] as an improved version of the deterministic policy gradient (DPG) algorithm that incorporates the best of DQL.

The major differences between RL approaches are inside the agents. Fig. 3 shows a representation of the DDPG agent. The elements that distinguish this algorithm the most with respect to other RL approaches are the following.

- Actor-critic: called this way due to the interactions between the value and the policy functions. The critic is the element that updates the value function according to the observed rewards. Meanwhile, the actor updates the policy function by learning the actions that maximize the value function. In an iterative-manner both converge to the optimal function approximations.

- ANNs for function approximation: both the value and the policy functions are represented by means of ANNs. This way the continuous action and state spaces can be efficiently handled. The actor network $\pi\left(\mathbf{s}, \boldsymbol{\vartheta}_{\pi}\right)$ is the approximation of the policy function and $\boldsymbol{\vartheta}_{\pi}$ is the set of parameters of the actor network to be trained. Similarly, $q\left(\mathbf{s}, \mathbf{a}, \boldsymbol{\vartheta}_{q}\right)$ is the approximation of the value function and $\vartheta_{q}$ is the set of parameters of the critic network to be trained.

- Target networks: the initial parameters of the actor and critic networks are copied into the target actor network $\pi\left(\mathbf{s}, \tilde{\boldsymbol{\vartheta}}_{\pi}\right)$ and target critic network $q\left(\mathbf{s}, \mathbf{a}, \tilde{\boldsymbol{\vartheta}}_{q}\right)$, respectively. These target networks are used for calculating the target values and their network parameters $\left(\tilde{\boldsymbol{\vartheta}}_{\pi}\right.$ and $\left.\tilde{\boldsymbol{\vartheta}}_{q}\right)$ are updated in a soft-manner to slowly track the original networks. Using these target networks, it is possible to set stable targets in order to train the critic without divergence.

- Experience replay: the experience tuple at each time step $\mathbf{e}_{\mathrm{t}}$ is stored in a replay memory $\mathcal{R}$, such that $|\mathcal{R}|$ is the number of experience tuples stored in $\mathcal{R}$. This memory is accessed by sampling random mini-batches $\mathcal{B}$ of size $|\mathcal{B}|$ to update the weights of the actor and critic networks. Using the experience replay improves the stability of the updates since correlation between samples is eliminated. 


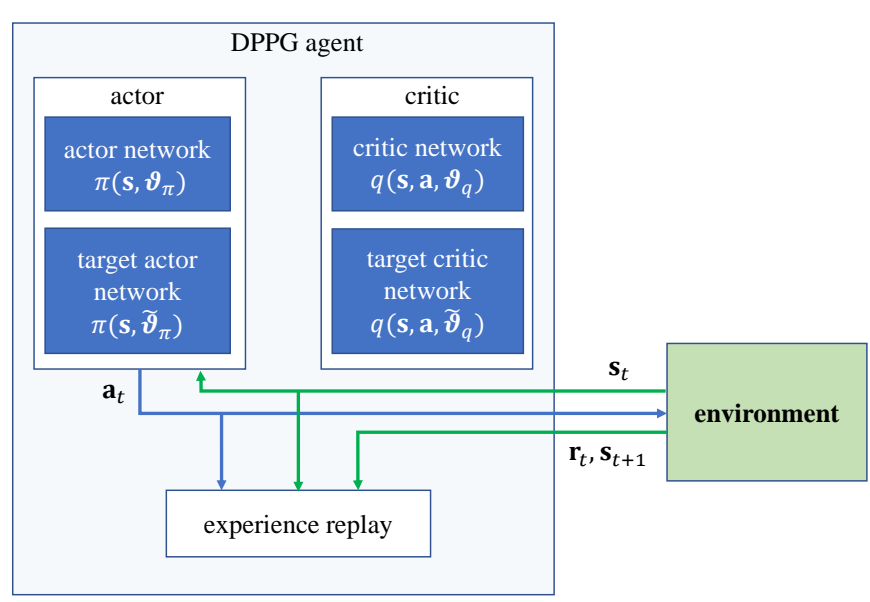

Fig. 3. Elements in the DDPG agent.

\section{Proposed Algorithm}

In this section, we present the DDPG-based algorithm that we propose to solve the optimization problem in (4). The values of the IRS phase-shift matrix $(\Theta)$ and the precoding matrix (P) that maximize the achievable sum-rate are calculated by using a DRL-based approach.

Algorithm 1 summarizes the steps of the proposed solution. We assume full CSI knowledge and, as a result, the channel matrices $\mathbf{H}$ and $\mathbf{H}_{\mathrm{IB}}$ are considered to be inputs of the algorithm. The other inputs are the user power constraint $T$, the long-term reward discount factor $\gamma$, and the target networks' updating rate $\tau$.

During the initialization stage, we create the actor and critic networks by randomly initializing the network parameters $\left(\boldsymbol{\vartheta}_{\pi}\right.$ and $\boldsymbol{\vartheta}_{q}$, respectively). The target actor and target critic networks are also created as exact copies of them. Hence, before the learning process starts, $\boldsymbol{\vartheta}_{\pi}=\tilde{\boldsymbol{\vartheta}}_{\pi}$ and $\boldsymbol{\vartheta}_{q}=\tilde{\boldsymbol{\vartheta}}_{q}$. During this stage, we also create the actor network optimizer and the critic network optimizer to later handle the updates of $\boldsymbol{\vartheta}_{\pi}$ and $\boldsymbol{\vartheta}_{q}$, respectively. The experience replay buffer $\mathcal{R}$ is created with no elements.

We propose to split the interactions between the DDPG agent and the environment into $E$ episodes with a defined finite number of steps $n_{s}$, since no terminal states can be defined in our problem. We have considered to set $n_{s}=200$ and to vary the number of episodes according to the intended number of interactions.

The initial state $\mathbf{s}_{0}$ is created as in (8), namely from randomly initialized $\mathbf{P}$ and $\Theta$ matrices. Starting from that state, the agent takes in every step the actions according to the actor network and an exploration noise $n_{\mathrm{e}} \sim \mathcal{N}\left(0, \sigma_{\mathrm{n}_{\mathrm{e}}}^{2}\right)$. The exploration noise improves the exploration efficiency by leveraging the off-policy behavior of the algorithm.

The learning stage (lines 12 to 21 in Algorithm 1) is first performed when there are enough stored experiences to sample a mini-batch of size $|\mathcal{B}|$. Notice that first mini-batches will be highly correlated since $|\mathcal{B}| \approx|\mathcal{R}|$. However, this effect soon disappears as $|\mathcal{R}|$ increases in every step.

At each step, the experience tuples in $\mathcal{B}$ are employed to train the actor and critic networks as described in lines from

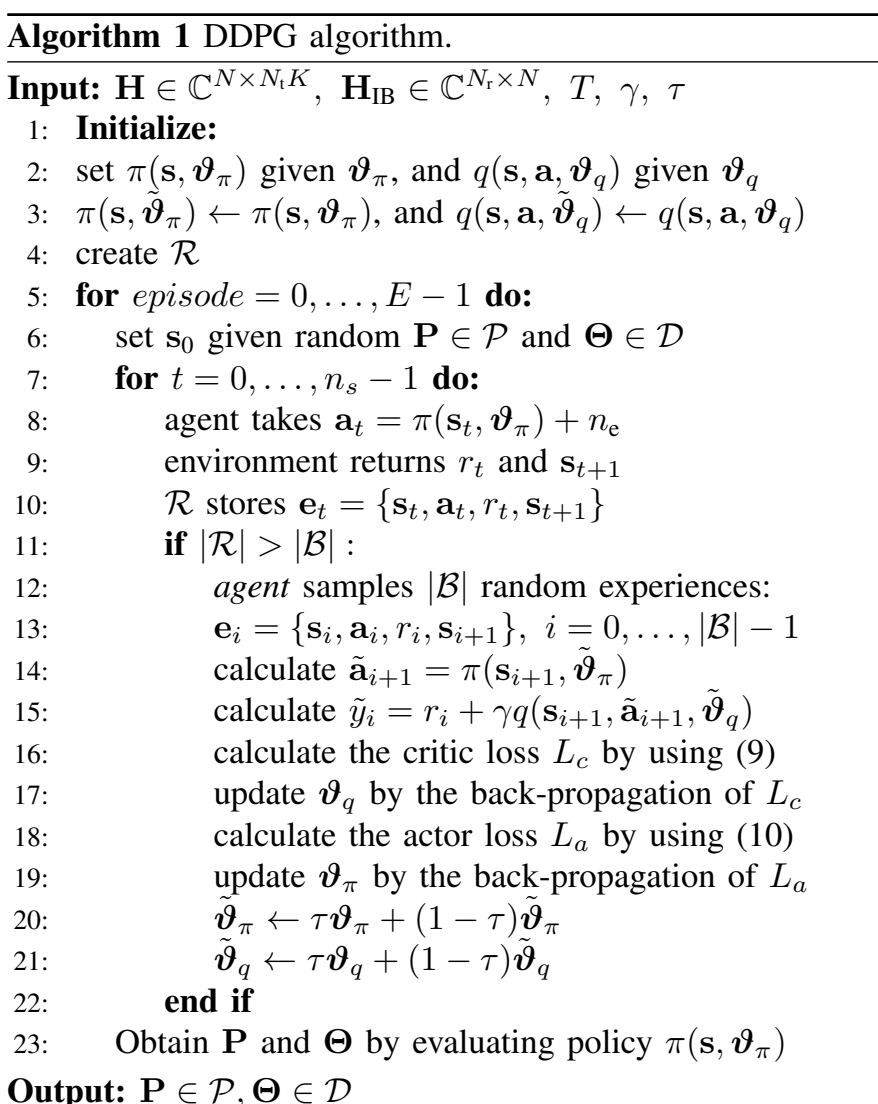

12 to 19 . As presented in [48], the target variables $\tilde{\mathbf{a}}_{i+1}$ and $\tilde{y}_{i}$ are calculated by means of the target actor network and the target critic network, respectively (lines 14 and 15). The critic loss, $L_{c}$, is next calculated as

$$
L_{c}=\frac{1}{|\mathcal{B}|} \sum_{i}\left(\tilde{y}-q\left(\mathbf{s}, \mathbf{a}, \vartheta_{q}\right)\right)^{2},
$$

and the obtained values are back-propagated by using the critic network optimizer. This way, the critic network is trained to minimize $L_{c}$ and to better represent the behavior of the actual action-value function.

On the other hand, the actor network is trained to predict the action that maximizes the output of the critic network. Because of this, the sign of the critic output value is changed, i.e.,

$$
L_{a}=-\frac{1}{|\mathcal{B}|} \sum_{i} q\left(\mathbf{s}, \pi\left(\mathbf{s}, \boldsymbol{\vartheta}_{\pi}\right), \boldsymbol{\vartheta}_{q}\right),
$$

and, hence, the back-propagation of the actor network optimizer can be perceived as a gradient ascent instead of descent. Notice that $L_{a}$ is intended only to update the actor network parameters. Thus, the actor network optimizer does not update the critic network parameters. To end the training stage, the actor target and the critic target networks are updated as described in the lines 20 and 21.

At the end of every learning episode, the policy is evaluated by running an evaluation episode. During this stage, we do not store the experiences nor update the networks. By following the actor network policy, the randomly initialized $\mathbf{P}$ and $\boldsymbol{\Theta}$ matrices are transformed to improve the sum-rate 


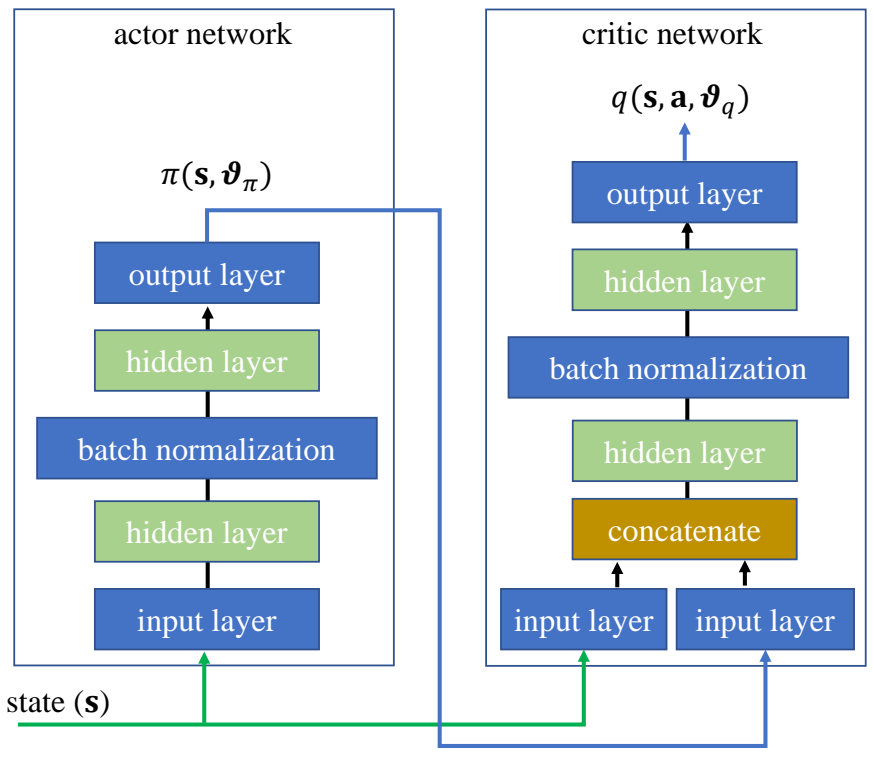

Fig. 4. Actor and critic network structures.

of the system. The matrices $\mathbf{P}$ and $\boldsymbol{\Theta}$ which provide the best performance are temporally stored, and returned as outputs at the end of the evaluation episode, if they outperform the previous output matrices.

\section{ANNs Structure}

The structures of the ANNs that we propose to use as actor and critic networks are presented in Fig. 4. As we said previously, the actor target and the critic target networks use the same structures.

The actor network input and output layer dimensions are equal to the state space dimension $D_{\text {state }}$ and the action space dimension $D_{\text {action }}$, respectively. Recall that $D_{\text {action }}=D_{\text {state }}$ and hence both layers have the same dimension in our specific configuration. The hidden layers are fully connect layers with $2 D_{\text {state }}$ neurons. There is not actually consensus about how many neurons should be included in hidden layers, but this value must be higher than the number of input features. Hence, we propose to use twice the dimension of the input. We made several tests with different configurations and no improvement was observed by increasing this number.

We used the rectified linear unit (ReLU) function as activation function at both hidden layers, but similar performance was also observed with the sigmoid function. At the output layer, we use the hyperbolic tangent (tanh) activation function to enforce the action values to lie in the interval $(-1,1)$ in order to improve the system stability.

The critic network input layer dimension is equal to $D_{\text {state }}+$ $D_{\text {action }}$ since it takes both state and action vectors as inputs. As shown in Fig. 4, these vectors are first treated independently and later concatenated inside the network. Hidden layers are also fully connected layers with $2\left(D_{\text {state }}+D_{\text {action }}\right)$ neurons. Similarly, we use the ReLU activation function in the hidden layers. The output layer dimension is one, since this network aims at predicting the value of the action-value function for
TABLE II

CONFIGURATION PARAMETERS

\begin{tabular}{|c||c|c|}
\hline Parameter & Description & Value \\
\hline$E$ & number of episodes & 50 \\
\hline$n_{s}$ & number of time steps per episode & 200 \\
\hline$|\mathcal{B}|$ & mini-batch size & 16 \\
\hline$|\mathcal{R}|$ & experience replay buffer size & 100000 \\
\hline$\mu_{a}$ & actor network optimizer learning rate & 0.001 \\
\hline$\mu_{c}$ & critic network optimizer learning rate & 0.001 \\
\hline$\tau$ & target network's updating rate & 0.005 \\
\hline$\gamma$ & discount factor & 0.99 \\
\hline
\end{tabular}

given state and action. Hence, we use the linear activation function at this output layer.

As proposed in [48], [51], [54], we include batch normalization layers in both actor and critic networks to improve the learning performance. By inserting batch normalization layers, higher learning rates can be considered and the network initialization values have lower impact in the network performance. We use the Adam optimizer in both networks since this algorithm has been proved to be computationally efficient and robust for supervised and deep reinforcement learning problems [50], [51], [55].

\section{Simulation Results}

In this section, we present results that validate the use of the DDPG framework to jointly optimize the IRS phase-shift and precoding matrices in IRS-assisted MU-MIMO communications. We consider the framework configuration as presented in previous subsections and follow the steps described in Algorithm 1.

Table II shows the configuration parameters employed in the computer experiments. For the sake of fairness, we have selected the number of episodes and steps per episode such that the running times of our proposal and the benchmark algorithms remain similar. Mini-batches with 16 elements set an adequate trade-off between learning performance and complexity. We create the experience replay buffer large enough to store all the experiences to be observed during a simulation running. The learning rate, updating rate and discount factor values have been chosen experimentally to provide the best performance in terms of system sum-rate.

We have selected two different model-driven approaches to be used as benchmarks. The first scheme is termed Alternating-ProG and uses a MMSE-based alternating minimization projected gradient algorithm to optimize the IRS and the user precoding matrices [56], [57]. The other is termed as ProG-MRT and is a baseline strategy where the IRS phaseshift matrix is optimized by means of a projected gradient algorithm and the precoding matrix is designed by following the maximum ratio transmitter (MRT) approach.

\section{A. Single-stream scenario}

Let us start considering an IRS-assisted single-stream MU-MIMO scenario as a specific case within the MS scenarios. All the previously discussed equations and methods are directly applicable to this case by only considering $N_{\mathrm{s}}=1$. 


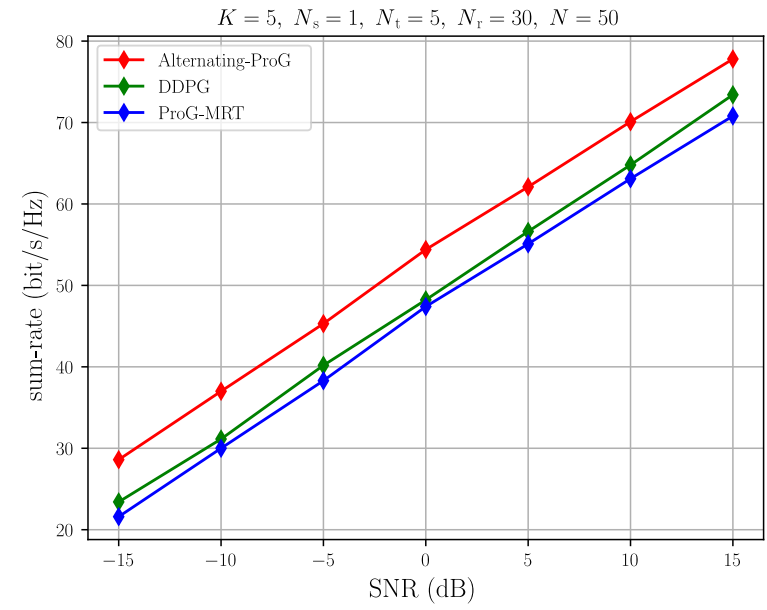

Fig. 5. Sum-rate $(\mathrm{bit} / \mathrm{s} / \mathrm{Hz})$ vs $\mathrm{SNR}(\mathrm{dB})$ for $K=5, N_{\mathrm{s}}=1, N_{\mathrm{t}}=$ $5, N_{\mathrm{r}}=30, N=50$.

Notice that, in this case, the precoding matrices $\mathbf{P}_{k} \in \mathbb{C}^{N_{\mathrm{t}} \times N_{\mathrm{s}}}$ will be precoding vectors $\mathbf{p}_{k} \in \mathbb{C}^{N_{\mathrm{t}} \times 1}$ instead.

Fig. 5 shows the achievable sum-rate obtained with the proposed DRL-based DDPG approach and the two benchmarks Alternating-ProG and ProG-MRT in a setup with $K=5$ users having $N_{\mathrm{t}}=5$ antennas each, an IRS with $N=50$ elements and a BS with $N_{\mathrm{r}}=30$ receiving antennas. In these simulation conditions, the Alternating-ProG algorithm outperforms our DRL-based approach, although our proposed scheme does slightly outperform the ProG-MRT one. This is an expected result that is explained from two important remarks. First, as stated in [20], the performance of linear MMSE precoders is near optimal when $N_{\mathrm{r}}>>K N_{\mathrm{s}}$ as occurs in this configuration. Although the necessary alternating procedure imposes limitations to its performance, we can say that the communication configuration is favorable for this approach. Second, the running times for all the algorithms are constrained to be similar. Hence, the capability of continuous improvement of the DRL-based proposed approach is not being fully leveraged. The performance of the proposed DDPG algorithm is expected to continuously improve by interacting with the environment in an online fashion.

The results shown in Fig. 6 allow us to better understand how the number of users affects the performance of the proposed approach and the benchmarks. Since the AlternatingProG benchmark outperforms the ProG-MRT algorithm in every scenario, we only consider the former for the ensuing analysis. The results in Fig. 6 consider a setup with $K$ users having $N_{\mathrm{t}}=2$ antennas each, an IRS with $N=50$ elements and a BS with $N_{\mathrm{r}}=2$ receiving antennas. When considering $K=2$, the behavior of both algorithms is similar to that previously observed. The Alternating-ProG benchmark performs better as long as it has enough degrees of freedom (DoF) to manage the inter-user interference (i.e., $\left.N_{\mathrm{r}} / K N_{\mathrm{s}} \geq 1\right)$. However, when this condition is not met (i.e., $N_{\mathrm{r}} / K N_{\mathrm{s}}<1$ ), the DRL-based DDPG proposal handles the interference significantly better and gets higher sum-rate values. Although the proposed scheme is also affected by the

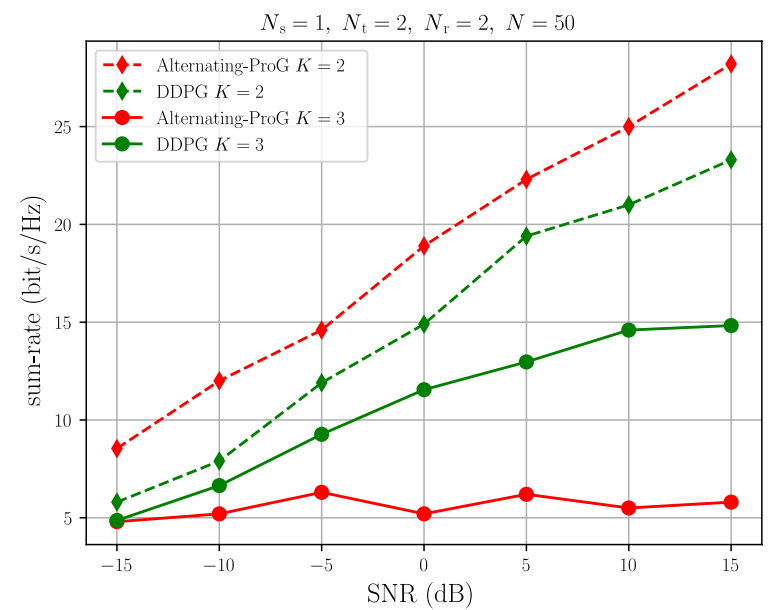

Fig. 6. Sum-rate (bit/s/Hz) vs SNR (dB) for $K \in\{2,3\}, N_{\mathrm{s}}=1, N_{\mathrm{t}}=$ $2, N_{\mathrm{r}}=2, N=50$.

inter-user interference, it provides almost three times the sumrate value obtained by the Alternating-ProG approach when $\mathrm{SNR}=15 \mathrm{~dB}$.

\section{B. Multi-stream}

Let us now consider an IRS-assisted MS MU-MIMO scenario as described in Section III. The achievable sum-rate values shown in Fig. 7 are obtained by considering $K=10$ users employing their $N_{\mathrm{t}}=2$ antennas to send $N_{\mathrm{s}}=2$ data streams each, an IRS with $N=50$ elements and a BS with $N_{\mathrm{r}}$ receiving antennas.

We address two different scenarios according to the number of receiving antennas at the BS, namely $N_{\mathrm{r}}=16$ and $N_{\mathrm{r}}=8$. It can be observed that $N_{\mathrm{r}} / K N_{\mathrm{s}}<1$ in both scenarios, which implies that the communication system has no enough DoF to handle the inter-user interference. In the first case with $N_{\mathrm{r}}=16$, the number of data streams managed is not much higher than $N_{\mathrm{r}}$ and the behavior of the algorithms is similar to that previously shown. In the low SNR regime $(\mathrm{SNR}<-5 \mathrm{~dB})$, benchmark algorithms slightly outperform our proposal. This is reasonable as in this SNR regime, the system performance is more sensitive to the channel noise than to the inter-user interference. However, as the SNR increases, these benchmark algorithms are not able to efficiently handle the inter-user interference and the sum-rate values decrease or remain constant. On the other hand, the proposed DDPGbased scheme manages the interference in a better way and the sum-rate values increase almost linearly with the SNR. The achievable sum-rate obtained by the DDPG-based proposal when $\mathrm{SNR}=15 \mathrm{~dB}$ is more than $40 \mathrm{bits} / \mathrm{s} / \mathrm{Hz}$ higher than both benchmark algorithms.

The second scenario configuration shown in Fig. 7 is a critic condition where the number of data streams to manage is higher than twice the number of receiving antennas. Under this condition, none of the algorithms is able to manage the interuser interference and a scheduling stage should be considered.

In the last experiment, we deeply analyze the impact of the relationship between the receiving antennas and transmitted 


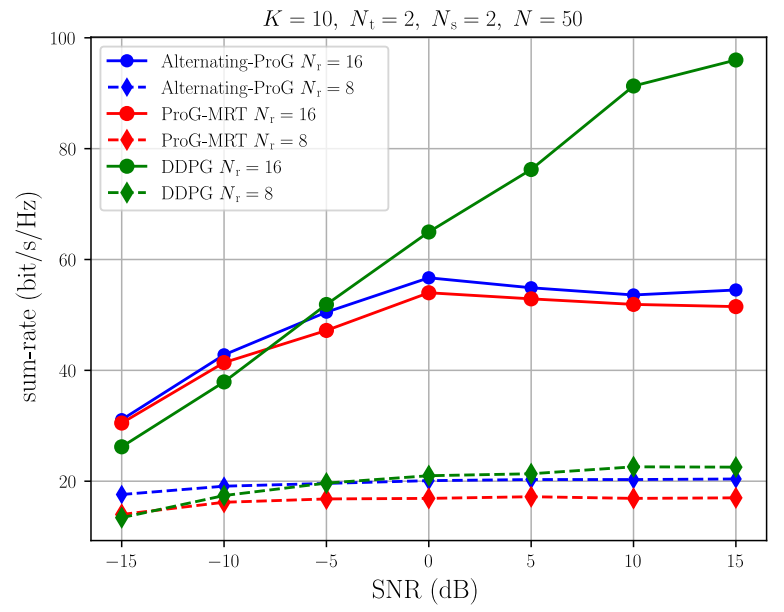

Fig. 7. Sum-rate (bit/s/Hz) vs SNR (dB) for $N_{\mathrm{r}} \in\{8,16\}, K=10, N_{\mathrm{t}}=$ $2, N_{\mathrm{s}}=2, N=50$.

data streams on the performance of the different algorithms. Fig. 8 plots the achievable sum-rate values by considering a MS scenario with $K=10, N_{\mathrm{s}}=2, N_{\mathrm{t}}=2, N=50, \mathrm{SNR}=$ $15 \mathrm{~dB}$ and $N_{\mathrm{r}}$ values that range from 8 to 30 . In this figure, we can observe how the performances of the Alternating-ProG benchmark and the proposed approach behave under different $N_{\mathrm{r}} / K N_{\mathrm{s}}$ ratio conditions. In the ensuing analysis we consider three main working regimes: $N_{\mathrm{r}} / K N_{\mathrm{s}}<<1, N_{\mathrm{r}} / K N_{\mathrm{s}}<1$, and $N_{\mathrm{r}} / K N_{\mathrm{s}} \geq 1$.

The first regime represents the critic scenario where the number of data streams to be managed is significantly higher than the number of receiving antennas at the BS. As previously discussed, none of the analyzed algorithms perform well in this regime and the sum-rate values remarkably degrade. In the third regime, both algorithms perform well although the Alternating-ProG benchmark slightly outperforms the proposed scheme. Recall that these results were obtained by considering similar running times and the ability for continuous improvement of the DDPG-based approach was not leveraged. The second regime is an intermediate scenario between the first and the third regimes where the proposed DDPG algorithm clearly outperforms the Alternating-ProG benchmark. When moving into this regime, the Alternating-ProG algorithm is not able to handle the interference between users. The sum-rate values increase very slowly by considering more receiving antennas, and no major performance improvement is observed until the third regime condition is met. On the other hand, the DDPG-based proposal manages the interference more efficiently and sum-rate values increase almost linearly by considering more receiving antennas. When $N_{\mathrm{r}}$ is reduced from 20 to 18 , the Alternating-ProG sum-rate value decreases near $75 \mathrm{bit} / \mathrm{s} / \mathrm{Hz}$, while this value only decreases about 25 $\mathrm{bit} / \mathrm{s} / \mathrm{Hz}$ if the DDPG-based proposal is considered. The results show that the sum-rate values achieved with the proposed scheme are at least $25 \mathrm{bit} / \mathrm{s} / \mathrm{Hz}$ higher when considering $N_{\mathrm{r}}$ values between 12 and 18 .

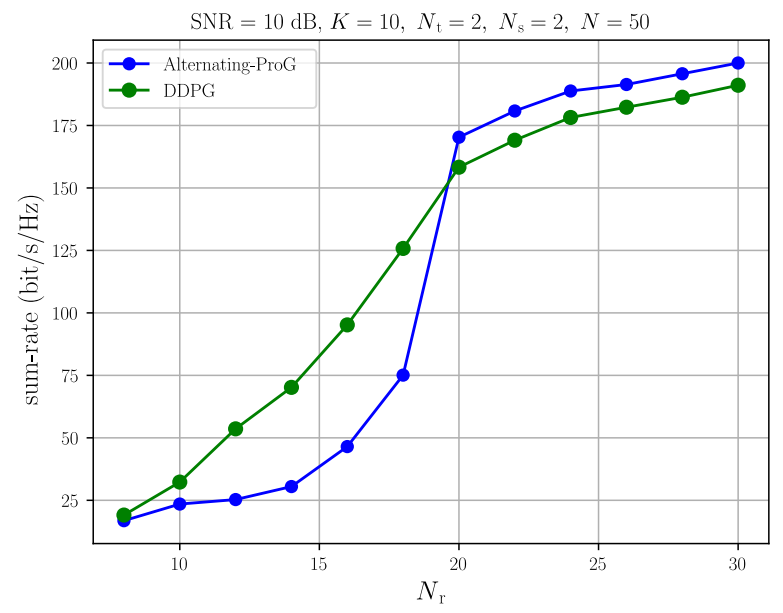

Fig. 8. Sum-rate (bit/s/Hz) vs $N_{\mathrm{r}}$ for $\mathrm{SNR}=10 \mathrm{~dB}, K=10, N_{\mathrm{t}}=2, N_{\mathrm{s}}=$ $2, N=50$.

TABLE III

COMPUTATIONAL COMPLEXITY

\begin{tabular}{|c|c|}
\hline Algorithm & Computational complexity order \\
\hline DDPG & $\mathcal{O}\left(N^{2}+K^{2} N_{\mathrm{t}}^{2} N_{\mathrm{s}}^{2}\right)$ \\
\hline Alternating-ProG & $\mathcal{O}\left(N K N_{\mathrm{t}} N_{\mathrm{s}} N_{\mathrm{r}} \epsilon\right)$ \\
\hline ProG-MRT & $\mathcal{O}\left(N K N_{\mathrm{t}} N_{\mathrm{s}} N_{\mathrm{r}} \epsilon\right)$ \\
\hline
\end{tabular}

\section{Computational Complexity}

As in [44] and [50], the computational complexity of the proposed DDPG-based algorithm is addressed by only considering the operational stage, i.e., the stage where the trained networks predict the matrices. The proposed algorithm learns in an online fashion and previous training is useful as long as no major changes occur in the communication configuration. Therefore, the computational complexity is determined by the structure of the actor network. For ANNs with fully connected layers, the computational complexity is bounded by $\mathcal{O}\left(\psi \zeta^{2}\right)$, where $\psi$ is the number of hidden layers and $\zeta$ is the number of neurons in the widest layer. We discard the number of layers because it is not related to the communication parameters. As we said previously, the number of neurons must be higher than the number of input features and this value is equal to $D_{\text {state }}+D_{\text {action }}$. Hence the computational complexity is in the order of $\mathcal{O}\left(N^{2}+K^{2} N_{\mathrm{t}}^{2} N_{\mathrm{s}}^{2}\right)$.

Table III shows the computational complexity order for the proposed DDPG algorithm and the benchmark algorithms. In both benchmark algorithms, the computational complexity factor is mostly related to the calculation of the gradients of the matrices. Notice that these steps are performed $\epsilon$ times at most. Unlike the benchmark algorithms, the complexity of the proposed solution is not related to $N_{\mathrm{r}}$ and it is less affected by the increase of $N$ because this element is out of the main multiplication term. These are major improvements, since performance in IRS-assisted MIMO communications is highly related to the number of IRS elements and the number of antennas in the BS. Hence, the proposed algorithm will scale better than the benchmarks. 


\section{CONCLUSION}

We have investigated a DRL-based approach for the joint optimization of the IRS phase-shift matrix and the user precoding matrices in the uplink of an IRS-assisted MS MUMIMO system. We selected the DDPG framework because it has proved to be effective when dealing with problems where the action and state spaces are continuous. By considering the pros and cons of related works, we propose an innovative configuration of the state and action spaces in which current matrix entries are the states and the variations in these values are the actions. The optimization problem is defined as a sumrate maximization. Hence, the sum-rate values are employed to calculate the reward function.

The validation results show that the DDPG algorithm is an effective alternative to solve the optimization problem considered. The proposed algorithm performance is similar to state-of-the-art algorithms when $N_{\mathrm{r}} \geq K N_{\mathrm{s}}$, and it handles the inter-user interference more efficiently when $N_{\mathrm{r}}<K N_{\mathrm{s}}$. Hence, it represents a more general solution for a larger variety of IRS-aided communication scenarios. In addition, the computational complexity of the proposed solution scales better than benchmark algorithms because it does not depend on the number of BS receiving antennas and is less affected by the increase in the number of IRS elements.

\section{ACKNOWLEDGMENTS}

This work has been funded by the Xunta de Galicia (by grant ED431C 2020/15, and grant ED431G2019/01 to support the CITIC, Centre for Information and Communications Technology Research, from the University System of Galicia), the Agencia Estatal de Investigación of Spain (by grants RED2018-102668-T and PID2019-104958RB-C42) and ERDF funds of the EU (FEDER Galicia 2014-2020 \& AEI/FEDER Programs, UE), and the predoctoral grant BES2017-081955.

\section{REFERENCES}

[1] S. Gong, X. Lu, D. T. Hoang, D. Niyato, L. Shu, D. I. Kim, and Y.-C. Liang, "Toward Smart Wireless Communications via Intelligent Reflecting Surfaces: A Contemporary Survey," IEEE Commun. Surveys Tuts., vol. 22, no. 4, pp. 2283-2314, 2020.

[2] X. Guan, Q. Wu, and R. Zhang, "Joint Power Control and Passive Beamforming in IRS-Assisted Spectrum Sharing," IEEE Commun. Lett., vol. 24, no. 7, pp. 1553-1557, Jul. 2020.

[3] E. Castañeda, A. Silva, A. Gameiro, and M. Kountouris, "An Overview on Resource Allocation Techniques for Multi-User MIMO Systems," IEEE Commun. Surveys Tuts., vol. 19, no. 1, pp. 239-284, 2017.

[4] J. Navarro-Ortiz, P. Romero-Diaz, S. Sendra, P. Ameigeiras, J. J. RamosMunoz, and J. M. Lopez-Soler, "A Survey on 5G Usage Scenarios and Traffic Models," IEEE Commun. Surveys Tuts., vol. 22, no. 2, pp. 905 929, 2020.

[5] L. Zhao, H. Zhao, K. Zheng, and W. Xiang, Massive MIMO in 5G Networks: Selected Applications, ser. SpringerBriefs in Electrical and Computer Engineering. Cham: Springer International Publishing, 2018. [Online]. Available: http://link.springer.com/10.1007/ 978-3-319-68409-3

[6] D. Pérez-Adán, O. Fresnedo, J. P. González-Coma, and L. Castedo, "Intelligent Reflective Surfaces for Wireless Networks: An Overview of Applications, Approached Issues, and Open Problems," Electronics, vol. 10, no. 19, p. 2345, 2021. [Online]. Available: https://www.mdpi. com/2079-9292/10/19/2345
[7] C. Pan, H. Ren, K. Wang, W. Xu, M. Elkashlan, A. Nallanathan, and L. Hanzo, "Multicell MIMO Communications Relying on Intelligent Reflecting Surfaces," IEEE Trans. Wireless Commun., vol. 19, no. 8 , pp. 5218-5233, Aug. 2020.

[8] C. Huang, A. Zappone, G. C. Alexandropoulos, M. Debbah, and C. Yuen, "Reconfigurable Intelligent Surfaces for Energy Efficiency in Wireless Communication," IEEE Trans. Wireless Commun., vol. 18 , no. 8, pp. 4157-4170, Aug. 2019.

[9] H. Guo, Y.-C. Liang, J. Chen, and E. G. Larsson, "Weighted Sum-Rate Maximization for Reconfigurable Intelligent Surface Aided Wireless Networks," IEEE Trans. Wireless Commun., vol. 19, no. 5, pp. 3064 3076, May 2020.

[10] G. Geraci, A. Garcia-Rodriguez, M. M. Azari, A. Lozano, M. Mezzavilla, S. Chatzinotas, Y. Chen, S. Rangan, and M. Di Renzo, "What Will the Future of UAV Cellular Communications Be? A Flight from 5G to 6G," arXiv:2105.04842 [cs, eess, math], May 2021. [Online]. Available: http://arxiv.org/abs/2105.04842

[11] Z. Wei, Y. Cai, Z. Sun, D. W. K. Ng, J. Yuan, M. Zhou, and L. Sun, "Sum-Rate Maximization for IRS-Assisted UAV OFDMA Communication Systems," IEEE Trans. Wireless Commun., vol. 20, no. 4, pp. 2530-2550, Apr. 2021.

[12] H. Shen, W. Xu, S. Gong, Z. He, and C. Zhao, "Secrecy Rate Maximization for Intelligent Reflecting Surface Assisted Multi-Antenna Communications," IEEE Commun. Lett., vol. 23, no. 9, pp. 1488-1492, Sep. 2019

[13] N. Hehao and L. Ni, "Intelligent Reflect Surface Aided Secure Transmission in MIMO Channel With SWIPT," IEEE Access, vol. 8, pp. 192 132-192 140, 2020.

[14] T. Bai, C. Pan, H. Ren, Y. Deng, M. Elkashlan, and A. Nallanathan, "Resource Allocation for Intelligent Reflecting Surface Aided Wireless Powered Mobile Edge Computing in OFDM Systems," IEEE Trans. Wireless Commun., vol. 20, no. 8, pp. 5389-5407, Aug. 2021.

[15] T. Bai, C. Pan, Y. Deng, M. Elkashlan, A. Nallanathan, and L. Hanzo, "Latency Minimization for Intelligent Reflecting Surface Aided Mobile Edge Computing," IEEE J. Sel. Areas Commun., vol. 38, no. 11, pp. 2666-2682, Nov. 2020

[16] S. Mao, N. Zhang, L. Liu, J. Wu, M. Dong, K. Ota, T. Liu, and D. Wu, "Computation Rate Maximization for Intelligent Reflecting Surface Enhanced Wireless Powered Mobile Edge Computing Networks," IEEE Trans. Veh. Technol., vol. 70, no. 10, pp. 10820-10 831, Oct. 2021.

[17] Z. Chu, Z. Zhu, X. Li, F. Zhou, L. Zhen, and N. Al-Dhahir, "Resource Allocation for IRS Assisted Wireless Powered FDMA IoT Networks," IEEE Internet Things J., pp. 1-1, 2021.

[18] Z. Chen, X. Ma, C. Han, and Q. Wen, "Towards intelligent reflecting surface empowered 6G terahertz communications: A survey," China Commun., vol. 18, no. 5, pp. 93-119, May 2021.

[19] X. Ma, Z. Chen, W. Chen, Z. Li, Y. Chi, C. Han, and S. Li, "Joint Channel Estimation and Data Rate Maximization for Intelligent Reflecting Surface Assisted Terahertz MIMO Communication Systems," IEEE Access, vol. 8, pp. 99565-99 581, 2020.

[20] E. Björnson, J. Hoydis, and L. Sanguinetti, "Massive MIMO Networks: Spectral, Energy, and Hardware Efficiency," FNT in Signal Processing, vol. 11, no. 3-4, pp. 154-655, 2017. [Online]. Available: http://www.nowpublishers.com/article/Details/SIG-093

[21] M. Hong and Z.-Q. Luo, "Signal Processing and Optimal Resource Allocation for the Interference Channel," arXiv:1206.5144 [cs, math], Jun. 2012. [Online]. Available: http://arxiv.org/abs/1206.5144

[22] W. Wang, X. Liu, J. Tang, N. Zhao, Y. Chen, Z. Ding, and $\mathrm{X}$. Wang, "Beamforming and Jamming Optimization for IRS-Aided Secure NOMA Networks," IEEE Trans. Wireless Commun., pp. 1-1, 2021.

[23] H. Ur Rehman, F. Bellili, A. Mezghani, and E. Hossain, "Joint Active and Passive Beamforming Design for IRS-Assisted Multi-User MIMO Systems: A VAMP-Based Approach," IEEE Trans. Commun., vol. 69, no. 10 , pp. 6734-6749, Oct. 2021.

[24] D. Chang, H. Jiang, J. Zhou, H. Zhang, and M. Mukherjee, "Capacity optimization using augmented lagrange method in intelligent reflecting surface-based MIMO communication systems," China Commun., vol. 17, no. 12, pp. 123-138, Dec. 2020.

[25] K. Zhang, C. Liu, H. Wang, and Y. Song, "An IRS-Aided mmWave Massive MIMO Systems Based on Genetic Algorithm," in 2020 IEEE 20th International Conference on Communication Technology (ICCT), Oct. 2020, pp. 288-293, iSSN: 2576-7828.

[26] V. P. Tuan and I. P. Hong, "Secrecy Performance Analysis and Optimization of Intelligent Reflecting Surface-Aided Indoor Wireless Communications," IEEE Access, vol. 8, pp. 109440-109452, 2020. 
[27] N. K. Kundu and M. R. McKay, "A Deep Learning-Based Channel Estimation Approach for MISO Communications with Large Intelligent Surfaces," in 2020 IEEE 31st Annual International Symposium on Personal, Indoor and Mobile Radio Communications, Aug. 2020, pp. 1-6, iSSN: 2166-9589.

[28] S. Liu, Z. Gao, J. Zhang, M. D. Renzo, and M.-S. Alouini, "Deep Denoising Neural Network Assisted Compressive Channel Estimation for mmWave Intelligent Reflecting Surfaces," IEEE Trans. Veh. Technol., vol. 69 , no. 8, pp. 9223-9228, Aug. 2020.

[29] A. M. Elbir, A. Papazafeiropoulos, P. Kourtessis, and S. Chatzinotas, "Deep Channel Learning for Large Intelligent Surfaces Aided mm-Wave Massive MIMO Systems," IEEE Wireless Commun. Lett., vol. 9, no. 9, pp. 1447-1451, Sep. 2020.

[30] N. K. Kundu and M. R. McKay, "Channel Estimation for Reconfigurable Intelligent Surface Aided MISO Communications: From LMMSE to Deep Learning Solutions," IEEE open j. Commun. Soc., vol. 2, pp. 471487, 2021

[31] L. Ge, Y. Guo, Y. Zhang, G. Chen, J. Wang, B. Dai, M. Li, and T. Jiang, "Deep Neural Network Based Channel Estimation for Massive MIMOOFDM Systems With Imperfect Channel State Information," IEEE Syst. J., pp. 1-11, 2021.

[32] H. Ye, G. Y. Li, and B.-H. Juang, "Power of Deep Learning for Channel Estimation and Signal Detection in OFDM Systems," IEEE Wireless Commun. Lett., vol. 7, no. 1, pp. 114-117, Feb. 2018.

[33] S. Yin, Y. Li, Y. Tian, and R. Yu, "Intelligent Reflecting Surface Enhanced Wireless Communications with Deep-learning-based Channel Prediction," IEEE Trans. Veh. Technol., pp. 1-1, 2021.

[34] W. Xia, G. Zheng, Y. Zhu, J. Zhang, J. Wang, and A. P. Petropulu, "A Deep Learning Framework for Optimization of MISO Downlink Beamforming," IEEE Trans. Commun., vol. 68, no. 3, pp. 1866-1880, Mar. 2020.

[35] W. Xia, G. Zheng, K.-K. Wong, and H. Zhu, "Model-Driven Beamforming Neural Networks," IEEE Wireless Commun., vol. 27, no. 1, pp. 68-75, Feb. 2020

[36] Y. Ge and J. Fan, "Beamforming Optimization for Intelligent Reflecting Surface Assisted MISO: A Deep Transfer Learning Approach," IEEE Trans. Veh. Technol., vol. 70, no. 4, pp. 3902-3907, Apr. 2021.

[37] I. Goodfellow, Y. Bengio, and A. Courville, Deep Learning. The MIT Press, 2016. [Online]. Available: http://www.deeplearningbook.org

[38] N. Zhao, Y.-C. Liang, D. Niyato, Y. Pei, M. Wu, and Y. Jiang, "Deep Reinforcement Learning for User Association and Resource Allocation in Heterogeneous Cellular Networks," IEEE Trans. Wireless Commun. vol. 18 , no. 11 , pp. 5141-5152, Nov. 2019.

[39] D. Silver, J. Schrittwieser, K. Simonyan, I. Antonoglou, A. Huang, A. Guez, T. Hubert, L. Baker, M. Lai, A. Bolton, Y. Chen, T. Lillicrap, F. Hui, L. Sifre, G. Driessche, T. Graepel, and D. Hassabis, "Mastering the game of Go without human knowledge," Nature, vol. 550, pp. 354 359, Oct. 2017.

[40] N. C. Luong, D. T. Hoang, S. Gong, D. Niyato, P. Wang, Y.-C. Liang, and D. I. Kim, "Applications of Deep Reinforcement Learning in Communications and Networking: A Survey," arXiv:1810.07862 [cs], Oct. 2018. [Online]. Available: http://arxiv.org/abs/1810.07862

[41] C. Huang, G. Chen, Y. Gong, M. Wen, and J. A. Chambers, "Deep Reinforcement Learning-Based Relay Selection in Intelligent Reflecting Surface Assisted Cooperative Networks," IEEE Wireless Commun. Lett., vol. 10, no. 5, pp. 1036-1040, May 2021.

[42] H. Yang, Z. Xiong, J. Zhao, D. Niyato, Q. Wu, H. V. Poor, and M. Tornatore, "Intelligent Reflecting Surface Assisted Anti-Jamming Communications: A Fast Reinforcement Learning Approach," IEEE Trans. Wireless Commun., vol. 20, no. 3, pp. 1963-1974, Mar. 2021.

[43] L. Jiao, P. Wang, A. Alipour-Fanid, H. Zeng, and K. Zeng, "Enabling Efficient Blockage-Aware Handover in RIS-Assisted mmWave Cellular Networks," IEEE Trans. Wireless Commun., pp. 1-1, 2021.

[44] Y. Zhao, I. G. Niemegeers, and S. M. H. De Groot, "Dynamic Power Allocation for Cell-Free Massive MIMO: Deep Reinforcement Learning Methods," IEEE Access, vol. 9, pp. 102 953-102 965, 2021.

[45] N. Nurani Krishnan, E. Torkildson, N. B. Mandayam, D. Raychaudhuri, E.-H. Rantala, and K. Doppler, "Optimizing Throughput Performance in Distributed MIMO Wi-Fi Networks Using Deep Reinforcement Learning," IEEE Trans. on Cogn. Commun. Netw., vol. 6, no. 1, pp. 135-150, Mar. 2020.

[46] R. S. Sutton and A. G. Barto, Reinforcement learning: an introduction, second edition ed., ser. Adaptive computation and machine learning series. Cambridge, Massachusetts: The MIT Press, 2018.

[47] A. Zai and B. Brown, Deep Reinforcement Learning in Action. New York, USA: Manning Publications Co., 2020.
[48] T. P. Lillicrap, J. J. Hunt, A. Pritzel, N. Heess, T. Erez, Y. Tassa, D. Silver, and D. Wierstra, "Continuous control with deep reinforcement learning," arXiv:1509.02971 [cs, stat], Jul. 2019. [Online]. Available: http://arxiv.org/abs/1509.02971

[49] F. B. Mismar, B. L. Evans, and A. Alkhateeb, "Deep Reinforcement Learning for 5G Networks: Joint Beamforming, Power Control, and Interference Coordination," IEEE Trans. Commun., vol. 68, no. 3, pp. 1581-1592, Mar. 2020.

[50] K. Feng, Q. Wang, X. Li, and C.-K. Wen, "Deep Reinforcement Learning Based Intelligent Reflecting Surface Optimization for MISO Communication Systems," IEEE Wireless Commun. Lett., vol. 9, no. 5, pp. 745-749, May 2020.

[51] C. Huang, R. Mo, and C. Yuen, "Reconfigurable Intelligent Surface Assisted Multiuser MISO Systems Exploiting Deep Reinforcement Learning," IEEE J. Sel. Areas Commun., vol. 38, no. 8, pp. 1839-1850, Aug. 2020.

[52] Z. Wang, L. Liu, and S. Cui, "Channel Estimation for Intelligent Reflecting Surface Assisted Multiuser Communications: Framework, Algorithms, and Analysis," IEEE Trans. Wireless Commun., vol. 19, no. 10 , pp. $6607-6620$, Oct. 2020.

[53] Q. Wu and R. Zhang, "Beamforming Optimization for Wireless Network Aided by Intelligent Reflecting Surface With Discrete Phase Shifts," IEEE Trans. Commun., vol. 68, no. 3, pp. 1838-1851, Mar. 2020.

[54] S. Ioffe and C. Szegedy, "Batch Normalization: Accelerating Deep Network Training by Reducing Internal Covariate Shift," arXiv:1502.03167 [cs], Mar. 2015. [Online]. Available: http://arxiv.org/ abs $/ 1502.03167$

[55] D. P. Kingma and J. Ba, "Adam: A Method for Stochastic Optimization," arXiv:1412.6980 [cs], Jan. 2017. [Online]. Available: http://arxiv.org/abs/1412.6980

[56] X. Yu, J.-C. Shen, J. Zhang, and K. B. Letaief, "Alternating Minimization Algorithms for Hybrid Precoding in Millimeter Wave MIMO Systems," IEEE J. Sel. Topics Signal Process., vol. 10, no. 3, pp. 485500, Apr. 2016.

[57] B. Ning, Z. Chen, W. Chen, and J. Fang, "Beamforming Optimization for Intelligent Reflecting Surface Assisted MIMO: A Sum-Path-Gain Maximization Approach," IEEE Wireless Commun. Lett., vol. 9, no. 7, pp. 1105-1109, Jul. 2020. 